\title{
DENTAL BIOMATERIALS IN NON-OPERATIVE MANAGEMENT OF DENTAL CARIES
}

\author{
Carounanidy Usha
}

\begin{abstract}
Dental caries is a disease that results in destruction of tooth structure called as carious lesions. The formation of carious lesion over a period of time is a sequel of alternating sequence of demineralization and remineralization of the hydroxyapatite crystals in the tooth structure. The yesteryears' management was focused on the lesions alone, in the surgical model of 'drill and fill'. Currently the disease prevention is receiving its due attention, a paradigm shift which is a result of a better and deeper understanding of the etiopathogenesis of carious disease. The formation of new lesions as well as remineralization of initial lesions are prevented by targeting various causative factors using biomaterials. These preventive materials and their current modifications are used in the non-operative treatment of caries, focusing on the preservation of the healthy tooth structure. This article highlights the latest advances in biomaterials used for the nonoperative treatment of dental caries.
\end{abstract}

Key words: Dental caries, remineralization, demineralization, carious lesions, non-operative treatment, operative treatment, dental biomaterials

\section{Introduction}

Dental Caries is a disease that results in tooth destruction called as carious lesion. It is defined as a disease that is characterised by the localized destruction of susceptible dental hard tissue by acidic by-products from bacterial fermentation of dietary carbobydrates. ${ }^{[1]}$ As evident by the definition, it is a multifactorial disease where multiple causes interact in a complex and dynamic manner. The biological factors that act in concert are the susceptible tooth structures, salivary factors, dietary sucrose and the cariogenic biofilm with predominance of Mutans streptococci.

Mutans streptococci in the dental biofilm, metabolize the dietary sucrose, which is an easily fermentable sugar, to release acidic by-products. This reduces the $\mathrm{pH}$ of the dental biofilm. In the acidic ambience minerals are dissolved from the tooth hydroxy apatite crystallites. Such a loss of calcified material from the tooth structure is called as demineralization. Continuous demineralization that occurs over time, on and within the tooth structure

can result in changes ranging from initial microscopic demineralization to macroscopic cavitation. These detectable changes that occur on the tooth are called as carious lesions.

But the demineralization process may not be continuous always. The unfavourable ambience and factors causing demineralization can be altered by beneficial salivary factors or by specific intervention by the operator, resulting in re-deposition of minerals to the tooth structure. This net gain of calcified material within the tooth structure, replacing that which was previously lost by demineralization is called as remineralization. ${ }^{[1]}$

The management of dental caries in the yester years focused only on the removal of carious lesions and restoration of the defect with a dental biomaterial. This was called as the surgical model, where drill and fill was the norm. Though the structure and function of the tooth was effectively restored, this method

* Professor, Department of Conservative Dentistry and Endodontics, Indira Gandhi Intitute Dental Science, Sri Balaji Vidyapeeth, Puducherry 607402, India. 
sacrificed precious healthy tooth structure also in the process of cavity preparation.

A restored tooth might also undergo repair or replacement throughout its life cycle, resulting in more and more loss of tooth structure, which can eventually lead to complete removal of the tooth. This is called as the restoration cycle. ${ }^{[2]}$ Albeit the significant improvements in the mechanical, chemical and biological properties of the restorative materials, the artificial materials, do not perform as effective as the natural enamel and dentin.

Thus with this understanding that tooth structure is a precious material to be preserved and also that the remineralization process is an inherent repair mechanism in the process of caries, the surgical model has weaned off to a large extent, reserved only for large cavitated lesions. The medical model of management has replaced the surgical model, where various causative factors are targeted using noninvasive strategies and materials, to prevent their interaction to cause dental caries.

Yet another factor contributing to this paradigm shift towards the medical model is the advancement in diagnosis and detection of dental caries at the very early incipient stages. Therefore the medical model is not only used in primary prevention of caries, but also in arresting the ongoing demineralization and remineralizing a new incipient lesion, called as secondary prevention. ${ }^{[3]}$

The medical model uses therapeutics that prevents or slows down demineralization and that which aids the remineralization process. This is done at all the causative levels such as at the microbial biofilm level, at the host/ tooth level, at the dietary level and at the salivary level.

\section{Non-operative therapeutic biomaterials that act at the tooth level}

The tooth-level treatments act by two ways in caries prevention; a. by increasing the tooth's resistance to demineralization $b$. By blocking the ecological niche on the tooth surface where the cariogenic organisms preferentially grow. Minerals such as fluorides and calcium-phosphate fortify the tooth against cariogenic acid attack. Laser ablation is another method that is being used to increase resistance of surface enamel to dissolution. The ecological niches are blocked by using pit and fissure sealants to prevent the proliferation of cariogenic organisms in this site.

\section{Fluorides}

The anticariogenic effect of fluoride is well established with robust scientific evidence. Formation of fluorapatite crystals that are more acid resistant than hydroxyapatite crystals has been attributed as the primary mechanism of action. ${ }^{[4]}$ The critical dissolution $\mathrm{pH}$ for fluorapatite is much lower than that for the hydroxy apatite crystals.

Systemic fluorides have been used largely to incorporate resistance to the tooth both in the preeruptive and post eruptive stage. But the presence of free fluoride ions in the biofilm and the oral fluid is recently considered to be more important than the concentration of bound fluoride in the enamel. It has been found that, under an acid attack, it is the free fluoride ions, along with the calcium ions, that influence the remineralization process. Thus emerged the wide spread use of topical fluorides, where free fluoride ions were made available at the tooth's vicinity in high concentration.

Fluoridated dentifrice and mouthwashes are the most common topical applications. But they do not ensure continuous availability of fluoride ions in the tooth vicinity. Therefore retention rate and sustained release of fluoride from the topical applications are considered imperative for prolonged action of fluoride. Gel and varnish as the modes of delivery provide better retention of fluoride over the enamel. Highest level of evidence is available on the superior anti-caries effect of both delivery methods. ${ }^{[5]}$

Sustained and controlled release of fluoride is being tried by the use of slow-release fluoride devices. [6] These devices are attached to the tooth surface by means of an adhesive. The fluoride from these reservoirs is then slowly released to the surrounding. A copolymer membrane device is available that is a small pellet made of a fluoride containing copolymer matrix surrounded by a rate controlling copolymer membrane. When the matrix gets hydrated, the fluoride dissolves and saturates the matrix. Thus, the ions move from saturated matrix to less saturated membrane and then to the saliva. This device can release $0.02-1 \mathrm{mg} \mathrm{F} / \mathrm{day}$, for up to 180 days. 
Glass beads that dissolve over a period of time, releasing fluoride, have also been evaluated. These beads can store fluoride in concentrations from $13.3 \%$ to $21.9 \%$ and release it even up to two years. [7]

Mixture of hydroxyapatite and sodium fluoride is under evaluation as a slow-release device. Brackets that are bonded to the tooth structure can hold a slow fluoride releasing pellet and it is claimed that they can be retained over 20 years also in the tooth. ${ }^{[8]}$ The fluoride releasing devices should also be able to recharge themselves. Polymerizable monomers containing chelating groups and fluoride-exchanging metal chelates that can "recharge" by taking up fluoride from fluoridated toothpaste or mouthwash is currently developed. ${ }^{[9]}$

\section{Casein phosphopeptide stabilized amorphous calcium phosphate (CPP-ACP):}

The fluoride uptake is influenced by concentration of calcium and phosphate ions in the saliva or biofilm. For every two fluoride ions, ten calcium ions and six phosphate ions are required to form one unit cell of fluorapatite (Ca10(PO4)6F2). Thus, topical applications of calcium and phosphate complexes are being used to enhance fluoride remineralization.

Unstabilized amorphous calcium phosphate (ACP), casein phosphopeptide stabilized amorphous calcium phosphate (CPP-ACP) and bioactive glass containing calcium sodium phosphosilicate are some of these systems available. ${ }^{[10]} \mathrm{CPP}-\mathrm{ACP}$ is available in dentifrice formulation, as a mouth rinse and as a non-sugar containing chewing gum. They are also available in combination with fluoride. The minerals are released under an acid challenge to supersaturate the saliva and help in remineralization. CPP-ACP binds strongly to hydroxyapatite. It increases the calcium phosphate level at the tooth biofilm interface. Thus it reduces the plaque $\mathrm{pH}$, as well as promote remineralization and prevent demineralization. They may be an important and effective adjunct to fluoride topical therapy in treatment of incipient lesions ${ }^{[11]}$

\section{Non-operative therapeutic biomaterials that act at the biofilm level}

Chemo prophylactic agents are used to modify the microbial colony in the dental biofilm. They act either by

1. Inhibiting initial adhesion/colonization
2. Inhibiting growth/metabolism

3. Disrupting mature biofilm.

Chemical agents such as chlorhexidine and triclosan are some of the commonly used caries prophylactic agents. The antimicrobial effect of an agent is determined by its potency, substantivity and bio availability. Bioavailability means the delivery of the agent to the intended site of action in a biologically active form and at effective doses. Topical application is the best method of delivery for oral antimicrobial agents to provide such bio availability. Substantivity means the agents' ability to bind to the oral surfaces and its subsequent rate of release form its binding sites. Thus an effective oral antimicrobial agent should be able to adhere to the mucosal, tooth pellicle surface or to the biofilm surface and provide its effect over a long period. The dose/ frequency of application of various agents depend on this property. For instance, a medication with low substantivity needs to be applied frequently to be bio available. Antimicrobials are available as mouth rinses, sprays, dentifrices, gels, varnishes and chewing gums/ lozenges.

\section{Chlorbexidine $(C H X)$}

Chlorhexidine is a bis-biguanide which is bactericidal and fungicidal. It is a broad spectrum agent targeting both the gram positive and gram negative organisms. Gram positive microbes such as Mutans streptococci are very sensitive to $\mathrm{CHX}$. The antibacterial effect of this medicament is attributed to the following:

1. It is a cationic agent that readily binds to negatively charged microbial surfaces. This results in cell wall disruption and cell death.

2. At high concentrations chlorhexidine it is bactericidal and in low concentrations bacteriostatic.

3. It inhibits the glucosyl transferase enzyme essential for microbial adhesion and the phosphoenolpyruvate phospho transferase essential for glucose transport across the cell membrane.

CHX is commonly available as $0.2 \%$ and 0.12 $\%$ solution / mouth rinse. Chlorhexidine has high substantivity which is a main reason for its superior antimicrobial effect. It is released in the mouth up to 8 hours after rinsing. Thus it is very effective as a topical chemo prophylactic agent.

However it does not specifically target the cariogenic organism. Long term use results in 
undesirable alteration in the entire oral microbial ecology. In addition there is no clinical correlation between its use and decline in caries incidence. Weighing its established side effects and the inconclusive evidence for benefits, the clinical usage of chlorhexidine is even being dissuaded ${ }^{[12]}$ However, 'inconclusive' evidence does not necessarily mean 'not effective'; therefore, the use of antimicrobials, in the current times, has been restricted to cariesactive individuals and to high caries risk individuals. Instead of prescribing it as a routine medication, it is prescribed as a regime for a short duration for a high risk individual, only if the risk assessment points toward a dominant microbial cause. ${ }^{[13]}$

Yet another common problem in the use of topical chemo prophylactic agent is the inability to maintain the minimum inhibitory concentration (MIC) of the drug in oral cavity. Chlorhexidine gels and chewing gums have been introduced to overcome this problem of retention. However these agents provide only a short term retention and sustenance of the MIC. Certain chewing gums have incorporated two types of antimicrobial agents which are released over a period of time and maintain the dosage above the MIC topically. Polymeric delivery systems have been patented where the agent is bonded to the copolymer, which hydrolyses to release the agent slowly into the oral cavity. ${ }^{[8]}$ Recently, a mineralbinding micellar drug delivery system was developed, which could quickly bind to the tooth surface and release encapsulated drug over a prolonged period of time. ${ }^{[14]}$

\section{Herbal interventions}

Owing to various challenges in the use of such potent synthetic agents, studies on the anticariogenicity of various plant products has increased in the last decade. The herbal interventions have shown efficacy in the inhibition of glucosyl transferases (GTFs) activity and insoluble glucan synthesis, inhibition of acid production from sucrose and inhibition of S. mutans adhesion on hard surface. However bactericidal effect has been found to be less in the herbal medicine. ${ }^{[15-21]}$ Scientifically established herbal extracts have been introduced in the tooth pastes and certain mouthwashes.

\section{Anti-microbial Peptides (AMP)}

Antimicrobial peptides are known for their broad spectrum antibacterial effect in the other fields of medicine. Recently, their use in targeting the Mutans streptococci has been investigated. They bind to the lipopolysaccharide of the microbial cell membrane, leading to cell death ${ }^{[22]}$. Recently an AMP is derived from milk protein that is suggested to be incorporated in the oral care products. ${ }^{[8]}$

But short half-life due to proteolytic degradation of the peptides is the current challenge in making it widely available for caries prevention. Target specific anti-microbial peptides are being developed which target only the mutans streptococci species. This is a promising concept in contrast to the other broad spectrum chemo prophylactic agents. ${ }^{[23]}$

\section{Probiotics and Replacement Therapy}

Instead of using chemicals, the micro-organism can be countered with another micro-organism. This is the concept behind the replacement therapy or the probiotic therapy. The disease causing 'wild strains' of bacteria can be displaced from their ecological niche, by introducing genetically modified form of the same organism, called as the 'effector strain'. These effector strains saturate these niches and prevent the colonization and multiplication of the wild strains again. A common example for a probiotic approach is the use of lactobacilli in intestinal infections. The mutans streptococci are genetically re-engineered to form strains that lack the inherent pathogenicity. One such strain developed lacks the enzyme lactate dehydrogenase, and so is incapable of producing lactic acid responsible for demineralization. Yet another genetically engineered streptococci that produces alkali instead of acid in the biofilm metabolism is under evaluation. ${ }^{[24]}$

\section{$\underline{\text { Vaccines }}$}

Passive immunization is currently being adopted in caries immunization. ${ }^{[25]}$ Instead of actively producing antibodies in the host system, ready-made antibodies to a specific antigen, produced elsewhere, is administered locally to obtain the desired result.

The antigen, when administered to a cow or a hen, stimulates the production of antibodies that are secreted in the milk and in the egg yolk, respectively. Recombinant monoclonal antibodies are also being used in passive immunization. All have shown a short-term reduction in the colonization of s. mutans in the rats and monkeys. 
Lately, the transgenic antibody from plants (tobacco and potato), also called as the 'plantibody', has been manufactured and is under a clinical trial. These antibodies are applied locally to the tooth, mostly in the form of mouth rinse.

However, only a transient drop in the microbial count lasting only for few hours does not satisfy the requirement of a vaccine, which is to provide a sustained availability of antibodies. This form of passive immunization is akin to any other mouth rinse, where lack of substantivity and retention might reduce the efficacy of the antibodies, necessitating frequent applications.

\section{Non-operative therapeutic biomaterials that act at the dietary/ salivary level}

Dietary sucrose is readily fermented by the Mutans streptococci, to release lactic acid that initiates the demineralization of the hydroxy apatite crystals. Drastically curtailing the sugar intake to prevent caries is not a prudent advice as it will yield poor patient compliance. Instead, sugar substitutes have been suggested. Sugar substitutes are of two types: 1 . Intense (non-caloric) sweeteners - e.g. saccharine and aspartame 2. Bulk (caloric) sweeteners - e.g. sorbitol and xylitol.

The anti cariogenicity of these sugars is due to the fact that these are not readily metabolised to acids by the cariogenic organisms. Some like sorbitol are metabolised very slowly. Xylitol possess antibacterial property as well, apart from being non-acidogenic. ${ }^{[26]}$

Sugar substitutes are added to the diet and beverages. But addition to the chewing gums is the common mode of delivery. The chewing gums also improve the salivary flow. With improved salivary flow, the other factors in saliva, such as the $\mathrm{pH}$ and buffer also improve, thus enhancing remineralization.

\section{$\underline{\text { Xylitol }}$}

Xylitol is a sugar alcohol with five carbon atoms, a pentitol. The anti-cariogenic property of this sugar has been attributed to the following:

1. Most oral streptococci do not ferment xylitol. Thus it is a non-acidogenic sugar

2. Entry of xylitol into the bacterial cell and accumulation as xylitol-5-phophate results in lysis of the cell. Thus it has a bacteriostatic action over the streptococcus mutans.।
3. It has inhibitory effect on glycolysis of streptococci.

4. It also reduces the adhesiveness of the bacteria by interfering in the polysaccharide formation.

Xylitol is mainly added in chewing gums. Along with salivary stimulation, it has been found to be effective in reducing caries. It is also available as candies. ${ }^{[27]}$ The recommended dosage is $6-10 \mathrm{gms} /$ day; 2 tabs of gum or two candies four times daily. However as has been discussed previously in this article, retention rate and constant availability of the medicament in the oral cavity is a concern with xylitol as well. Thus studies are focusing on novel delivery systems to improve the drug availability in the tooth vicinity, that too, on demand.

\section{Conclusion}

1. Dental caries is still evading the sincere attempts of science towards eradication. This is mainly due to the multifactorial and complex nature of the disease.

2. Though a plethora of preventive NOT strategies are available and are being well researched, a well-designed and custom made treatment plan is mandatory to take complete advantage of them.

3. Biomaterials used as topical agents constitute the majority of the NOT procedures. Innovative delivery modes and methods are the need of the hour to face the challenges regarding constant bio availability, retention rate and substantivity.

4. Role of genetics in the modification of the cariogenic biofilm dominates current research.

5. Fluoride is still considered as the gold standard in caries prevention through remineralization. Research is focusing on the identification of alternate to fluorides in the abundant plant products.

\section{References}

1. Longbottom C, Huysman MC, Pitts NB, Fontana M. Glossary of terms. In: Pitts NB, editor. Detection, assessment, diagnosis and monitoring of caries. Basel, Krager: Monogr Oral Sci; 2009. pp. 209-16.

2. Simonsen R J, Stallard R E. Sealant-restorations utilizing a dilute filled composite resin: one year results. Quintessence Int 1977;23: 307-315.

3. Steinberg SI. Understanding and managing dental caries: a medical approach. Alpha Omega 2007;100(3):127-34. 
4. SBU. Swedish Council on Technology assessment in health care. 2002. Prevention of dental caries: A systematic review. Rep No 161; pp. 1-26

5. American Dental Association Council on Scientific Affairs. Professionally applied topical fluoride: Evidence-based clinical recommendations.J Am Dent Assoc. 2006;137:11519.

6. Pessan JP, Al-Ibrahim NS, Buzalaf MA, Toumba KJ. Slowrelease fluoride devices: a literature review. J Appl Oral Sci. 2008;16:238-46.

7. Andreadis GA, Toumba KJ, Curzon ME. Slow-release fluoride glass devices: in vivo fluoride release and retention of the devices in children. Eur Arch Paediatr 2006;7(4):258-61.

8. Chen F, Wang D. Novel technologies for the prevention and treatment of dental caries: a patent survey. Expert Opin Ther Pat. 2010;20(5):681-94.

9. Bayrak S, Tunc ES, Aksoy A, Ertas E, Guvenc D, Ozer S. Fluoride Release and Recharge from Different Materials Used as Fissure Sealants. European Journal of Dentistry 2010;4(3):245-250.

10. Reynolds EC. Calcium phosphate-based remineralization systems: scientific evidence? Aust Dent J 2008; 53(3):268-73.

11. Azarpazhooh A, Limeback H. Clinical efficacy of casein derivatives. a systematic review of the literature. J Am Dent Assoc 2008;139:915-24.

12. Autio-Gold J. The role of chlorhexidine in caries prevention. Oper Dent 2008;33:710-6.

13. Anusavice KJ. Present and future approaches for the control of caries. J Dent Educ 2005;69:538-54.

14. Chen F, Liu XM, Rice KC, et al. Tooth-binding micelles for dental caries prevention. Antimicrob Agents Chemother 2009;53(11):4898-902.

15. Al-Hebshi NN, Nielsen O, Skaug N. In vitro effects of crude khat extracts on the growth, colonization, and glucosyltransferases of Streptococcus mutans. Acta Odontol Scand 2005;63(3):136-42.

16. Rahim ZH, Khan HB. Comparative studies on the effect of crude aqueous (CA) and solvent (CM) extracts of clove on the cariogenic properties of Streptococcus mutans. J Oral Sci 2006;48(3):117-23.

17. Koo H, Nino de Guzman P, Schobel BD, et al. Influence of cranberry juice on glucan-mediated processes involved in Streptococcus mutans biofilm development. Caries Res 2006;40(1):20-7.

18. Yu HH, Lee DH, Seo SJ, You YO. Anticariogenic properties of the extract of Cyperus rotundus. Am J Chin Med 2007;35:497-505
19. Brighenti FL, Luppens SB, Delbem AC, et al. Effect of Psidium cattleianum leaf extract on Streptococcus mutans viability, protein expression and acid production. Caries Res 2008;42(2):148-54.

20. Yu HH, Lee JS, Lee KH, et al. Saussurea lappa inhibits the growth, acid production, adhesion, and water-insoluble glucan synthesis of Streptococcus mutans. J Ethnopharmacol 2007;111:413-7.

21. Papetti A, Pruzzo C, Daglia M, et al. Effect of barley coffee on the adhesive properties of oral streptococci. J Agric Food Chem 2007;55(2):278-84.

22. Guggenheim B, Giertsen E, Schupbach P, Shapiro S. Validation of an in vitro biofilm model of supragingival plaque. J Dent Res 2001;80(1):363-70.

23. Eckert R, He J, Yarbrough DK, et al. Targeted killing of Streptococcus mutans by a pheromone-guided "smart" antimicrobial peptide. Antimicrob Agents Chemother 2006;50(11):3651-7.

24. Meurman JH, Stamatova I. Probiotics: contributions to oral health. Oral Dis 2007;13:443-51.

25. Abiko Y. Passive immunization against dental caries and periodontal disease: development of recombinant and human monoclonal antibody. Crit Rev Oral Biol Med 2000;11:14058.

26. Borges Yáñez SA. Sugar substitutes in the prevention of dental caries: review of the literature. Pract Odontol 1991;12(8):59-60.

27. Antonio AG, Pierro VS, Maia LC. Caries preventive effects of xylitol-based candies and lozenges: a systematic review. J Public Health Dent 2011;71(2):117-24. 